\title{
Prognostic model based on autophagy-related IncRNAs in gastric cancer
}

\section{Mengqiu Cheng}

Anhui Medical University

Wei Cao

Anhui Medical University

Bo Chen ( $\nabla$ chenbo831116@163.com )

First Affiliated Hospital of Anhui Medical University https://orcid.org/0000-0003-0524-1105

Primary research

Keywords: Gastric cancer, Prognosis, Autophagy, Nomogram

Posted Date: May 22nd, 2020

DOI: https://doi.org/10.21203/rs.3.rs-29662/v1

License: (1) This work is licensed under a Creative Commons Attribution 4.0 International License. Read Full License 


\section{Abstract}

Background: Gastric cancer (GC) is one of the most pravelent cancer in the world. Although increasing studies have indicated that autophagy-related long non-coding RNA (IncRNA) plays an essential role in the occurrence of $\mathrm{GC}$, the prognosis of GC based on autophagy is still deficient.

Method: Autophagy-related IncRNAs were obtained by using the correlation test with the autophagyrelated gene. Data was downloaded from The Cancer Genome of Atlas stomach adenocarcinoma (TCGASTAD) dataset. The prognostic autophagy-related IncRNAs significantly correlated with survival of TCGASTAD dataset were obtained by using Kaplan-Meier and univariate Cox regression analysis. TCGA-STAD dataset was separated into a training set and a testing set randomly. The model was constructed based on the training set through the least absolute shrinkage and selection operator (LASSO) regression. The testing set and TCGA-STAD were used to validate the accuracy of the model. Every patient got a risk score (RS) and patients were separate into high-risk group and low-risk group due to the median RS. The prognostic network was built and the mRNAs in the system were analyzed through Gene Ontology (GO) and Kyoto Encyclopedia of Genes and Genomes (KEGG) analysis. The signaling pathways that the differentially expressed genes (DEGs) between two types of risk group mainly participated in were distinguished through Gene Set Enrichment Analysis (GSEA). The individual's survival rate was predicted through the nomogram.

Results: 24 autophagy-related IncRNAs were found strongly associated with the survival of the TCGASTAD dataset. Among them, 11 IncRNAs were selected to build the risk score model through LASSO regression. The multivariate Cox analysis showed that the RS could be an independent prognosis predictor. The Kaplan-Meier survival analysis and the Receiver Operating Characteristic (ROC) curve indicated the model had an excellent prediction effect. GO, and KEGG analysis revealed that the mRNAs in the prognostic network were mainly involved in the autophagy and ubiquitin-like protein ligase binding. GSEA analysis uncovered that the DEGs in high-risk group partially participated in the ECM receptor interaction and other signaling pathways.

Conclusions: Our results indicated that the risk score model based on the autophagy-related IncRNAs performed well in the prediction of prognosis for patients with GC.

\section{Background}

Gastric cancer (GC) is the fifth most prevalent tumor and one of the deadliest carcinomas worldwide [1], among which $90 \%$ are adenocarcinomas [2]. Although there has been a stable decreasing in GC occurrence and mortality rates globally over several decades after the beginning of 19th century [3, 4], GC remains a high case fatality rate of $75 \%$ throughout the world [5]. Due to the insufficient understanding of the molecular mechanism [6] and the lack of relevant clinical prediction systems [7], most patients with GC have already been in the advanced stage when they are diagnosed [8], which brings great trouble to the patient's survival [9] and clinical therapy. Therefore, it is critical to construct an accuracy prediction 
system for GC, which have the ability for early diagnosis and prevent the disease before premalignant lesions have developed.

Autophagy is a highly conserved and evolutionarily ancient catabolic process which can degrade the misfolded proteins and damaged organelles [10]. For the past few years, knowledge about the function of autophagy has developed. It has been found that autophagy participated in a plenty of physiological processes in mammal, including quality control of proteins and organelles, immunity, nutrient deprivation, hypoxia, drug stimuli, stress, and prevention of neurodegeneration [11]. Autophagy can also regulate biological process including apoptosis, protein synthesis, cell growth and proliferation through the AMPK/mTOR pathway, PI3K/Akt/mTOR pathway, P53 pathway and other signaling pathways [12-14]. What' more, the role of autophagy in the progression of carcinoma also has several breakthroughs.

The effect of autophagy is considered controversial in tumorigenesis, which can both promote and suppress cancer development under different cell types or stress modes [15]. In general, it is thought that autophagy prevented carcinogenesis $[16,17]$. Nevertheless, once tumor is established, increased autophagic flux often promotes tumor cell growth by providing energy and vital compounds upon various stress stimuli [18]. Similarly, growing studies have also proved that autophagy had a significant effect on the GC.

The role of autophagy in GC is also complex and contradictory. Some researches supported that the autophagy was a tumor promoter in GC. Cause autophagy inhibitor will destroy the protective mechanism of GC cell and promotes cell death induced by therapeutic drugs [19-21]. However, PD-L1 expression was also found enhanced by autophagy inhibition in GC [22]. What's more, a study has shown that autophagy inducers such as AMPK inducers could be used in the GC treatment, which will lead to autophagic cell death of tumor cells [23].

In recent years, the relationship between long non-coding RNA (IncRNA) and autophagy has several breaks in the diagnosis, treatment, and prognosis of GC. Pieces of evidence showed that IncRNA is vital for the occurrence, prognosis, and chemoresistance of GC by regulating autophagy-related mRNA [2426]. Some studies have demonstrated that silencing of LINC01419 and CCAT2 promotes autophagy through constraining the PI3K/Akt1/mTOR pathway thus inhibiting the invasion and migration of GC cells [27, 28]; Another study also revealed that autophagy was associated with the proliferation of GC cells, partially due to the MALAT1 promoted by downregulating miR-204 [29]. However, most of these experiments only explored the role of one or a few IncRNAs in the autophagy of GC, and could not fully explain the relevant mechanisms.

To be concluded, the role of autophagy-related IncRNA in GC is complicated and controversial, which involves hundreds of molecular in this process. Therefore, a model consisted of multiple autophagyrelated IncRNAs will have a better prognosis predicting accuracy than the single. For this purpose, we built a risk score model based on the multiple autophagy-related IncRNAs, which also performed well in the prediction of prognosis for the GC patients in the clinic. 


\section{Materials And Methods}

\section{Screening for the autophagy-associated IncRNAs}

Autophagy-associated genes were obtained through the HADb: Human Autophagy Database website (http://www.autophagy.lu/). Autophagy-related IncRNAs were obtained by using the correlation test of these genes with $\mathrm{R}$ version 3.6.3 software (Pearson correlation coefficient $>0.3, P<0.001$ ).

\section{Data acquisition}

All 350 patients' data of STAD were downloaded from The Cancer Genome Atlas (TCGA) website (https://portal.gdc.cancer.gov/). All the data was submitted to R software and randomly separated into the training set and testing set.

\section{Identification of the prognostic autophagy-related IncRNAs}

Kaplan-Meier and univariate Cox regression analysis was applied to select the prognostic autophagyrelated IncRNA in TCGA-STAD dataset $(P<0.05)$.

\section{Survival analysis}

Least absolute shrinkage and selection operator (LASSO) regression was adopted to build the model using the R package "glmnet" with prognostic autophagy-related IncRNAs selected before. In this process, only the most potent prognostic markers were chosen and constituted the optimal panel of prognosis which achieved the best performance. Each patient got a risk score (RS) incorporating the expression of the IncRNAs $\left(\operatorname{Exp}_{i}\right)$ and the corresponding LASSO coefficients $($ Coef $)$, Risk Score $=\sum_{i=1}^{n} \operatorname{Exp}_{i}{ }^{*}$ Coef $_{i}$. The median RS was chosen to divide patients into high-risk group and low-risk group in the training set, testing set and TCGA-STAD dataset respectively. Multivariate Cox regression was used to assess whether the RS could be an independent predictor. Kaplan-Meier curves were used to check the significant difference in survival probability for patients between two types of risk group. An area under the Receiver Operating Characteristic (ROC) curve (AUC) was used to examine the accuracy. All statistics were processed through $\mathrm{R}$ version 3.6.3 software (https://www.r-project.org/) $(P<0.05)$.

\section{Network construction and pathway analysis}

The co-expressed mRNAs were obtained by using the correlation test with $\mathrm{R}$ software (Pearson correlation coefficient $>0.3, P<0.001)$. The network was constructed and visualized through Cytoscape software (https://cytoscape.org/). The Sankey diagram was built using the R software. The Gene Ontology (GO) and Kyoto Encyclopedia of Genes and Genomes (KEGG) analysis were performed using the "clusterProfiler" package of R software (Gene ratio $>0.05, P<0.05$ ). The signaling pathways that the differentially expressed genes (DEGs) mainly participated in between two types of risk group were distinguished through Gene Set Enrichment Analysis (GSEA). 
A nomogram was constructed through the "rms" package for R combining the risk type and other clinical features including age, gender, stage, and TNM stage to predict the individual's survival probability.

\section{Results}

\section{Characteristics of patients}

TCGA-STAD dataset was patients diagnosed with stomach adenocarcinoma, which consisted of a total of 350 patients. Kaplan-Meier survival curves were plotted for TCGA-STAD dataset regarding major clinical features (Supplementary Fig).

\section{Selecting of prognostic autophagy-related LncRNA for TCGA-STAD dataset}

The autophagy-related IncRNAs were obtained by using the correlation test of autophagy-related genes (Pearson correlation coefficient $>0.3, P<0.001$ ). A total of 24 autophagy-related IncRNAs were significantly correlated with the survival for TCGA-STAD through Kaplan-Meier and univariate Cox regression analysis $(P<0.05)$ (Fig. 1).

\section{Construction of prognostic risk score model based on autophagy-related IncRNAs}

All the patients from TCGA -STAD cohort were randomly separated into a training set and testing set $(P>$ 0.05) (Table 1). Then all these 24 identified prognostic LncRNAs were analyzed with the LASSO regression analysis (Fig. 2a) and 3-fold positive cross-validation (Fig. 2b) in the training set. The regression coefficient was computed to select the optimal panel of prognosis. The model achieved the best performance while 11 autophagy-related LncRNAs were included. Combining the expression of the autophagy-related IncRNAs and LASSO coefficients (Table 2), each patient got a RS as a measure of survival risk. 
Table 1

Major clinical characteristics for the STAD patients in the training set, testing set and TCGA-STAD dataset

\begin{tabular}{|c|c|c|c|}
\hline \multirow[t]{2}{*}{ Characteristics } & \multicolumn{2}{|l|}{ TCGA-STAD cohort } & \multirow[t]{2}{*}{$P$ value } \\
\hline & Training set $n=175(\%)$ & Testing set $n=175(\%)$ & \\
\hline Age & & & 0.1918 \\
\hline $30-50$ & 19(10.86\%) & $10(5.71 \%)$ & \\
\hline $50-70$ & $104(59.43 \%)$ & $96(54.86 \%)$ & \\
\hline $70-90$ & $52(29.71 \%)$ & $69(39.43 \%)$ & \\
\hline Gender & & & 0.3265 \\
\hline female & $62(35.43 \%)$ & $62(35.43 \%)$ & \\
\hline male & $113(64.57 \%)$ & $113(64.57 \%)$ & \\
\hline T stage & & & 0.909 \\
\hline T1-T2 & $37(21.14 \%)$ & $53(30.29 \%)$ & \\
\hline T3-T4 & 138(78.86\%) & $122(69.71 \%)$ & \\
\hline $\mathrm{N}$ stage & & & \\
\hline N1 & $49(28.00 \%)$ & $54(30.86 \%)$ & 0.6206 \\
\hline N2 & $44(25.14 \%)$ & $49(28.00 \%)$ & \\
\hline N3 & $35(20.00 \%)$ & $37(21.14 \%)$ & \\
\hline N4 & $47(26.86 \%)$ & $35(20.00 \%)$ & \\
\hline M stage & & & \\
\hline M0 & 156(89.14\%) & 154(88.00\%) & 0.2374 \\
\hline M1 & $19(10.86 \%)$ & $21(12.00 \%)$ & \\
\hline Clinical Stage & & & 0.6475 \\
\hline Stage I & $21(12.00 \%)$ & $26(14.86 \%)$ & \\
\hline Stage II & $50(28.57 \%)$ & $61(34.86 \%)$ & \\
\hline Stage III & $83(47.43 \%)$ & $63(36.00 \%)$ & \\
\hline Stage IV & $21(12.00 \%)$ & $25(14.29 \%)$ & \\
\hline Pathological Grade & & & 0.3813 \\
\hline
\end{tabular}




\begin{tabular}{|c|c|c|c|}
\hline \multirow[t]{2}{*}{ Characteristics } & \multicolumn{2}{|l|}{ TCGA-STAD cohort } & \multirow[t]{2}{*}{$P$ value } \\
\hline & Training set $n=175(\%)$ & Testing set $n=175(\%)$ & \\
\hline Grade 1 & $4(2.29 \%)$ & $5(2.86 \%)$ & \\
\hline Grade 2 & $54(30.86 \%)$ & $71(40.57 \%)$ & \\
\hline Grade 3 & 117(66.86\%) & $99(56.57 \%)$ & \\
\hline
\end{tabular}

Table 2

The IncRNAs in the risk score model were strongly associated with the survival in TCGA-STAD dataset

\begin{tabular}{|lllllllll|}
\hline \multirow{2}{*}{ IncRNA } & \multicolumn{3}{l}{ Kaplan-Meier analysis } & \multicolumn{3}{l}{ Univariate Cox regression analysis } & LASSO \\
\cline { 2 - 7 } & B & SE & P-value $^{*}$ & HR & HR.95L & HR.95H & P-value* $^{*}$ & \\
\hline AC092574.1 & -0.348 & 0.171 & 0.006 & 0.706 & 0.505 & 0.987 & 0.042 & -0.009 \\
\hline AL353804.1 & -0.279 & 0.131 & 0.002 & 0.757 & 0.585 & 0.979 & 0.034 & -0.238 \\
\hline AC005586.1 & -0.270 & 0.085 & 0.000 & 0.764 & 0.646 & 0.903 & 0.002 & -0.178 \\
\hline IP05P1 & -0.223 & 0.092 & 0.022 & 0.800 & 0.668 & 0.959 & 0.016 & -0.171 \\
\hline AL355574.1 & -0.207 & 0.076 & 0.033 & 0.813 & 0.701 & 0.943 & 0.006 & -0.087 \\
\hline AP003392.1 & -0.187 & 0.092 & 0.017 & 0.830 & 0.692 & 0.994 & 0.043 & -0.052 \\
\hline HAGLR & 0.081 & 0.030 & 0.001 & 1.084 & 1.023 & 1.149 & 0.007 & 0.065 \\
\hline LINC01705 & 0.092 & 0.041 & 0.039 & 1.097 & 1.012 & 1.188 & 0.024 & 0.027 \\
\hline AP001033.2 & 0.195 & 0.099 & 0.049 & 1.215 & 1.000 & 1.477 & 0.050 & 0.154 \\
\hline AC009948.1 & 0.197 & 0.079 & 0.041 & 1.218 & 1.043 & 1.423 & 0.013 & 0.252 \\
\hline AP001528.2 & 0.346 & 0.130 & 0.026 & 1.413 & 1.095 & 1.825 & 0.008 & 0.065 \\
\hline
\end{tabular}

HR, hazard ratio; HR.95L-HR.95H, 95\% confidential interval. ${ }^{*} P<0.05$

\section{Validation of the risk score model for survival prediction in the training set, testing set and TCGA-STAD dataset}

Based on the median RS, patients were divided into high-risk group and low-risk group. IncRNA AC005586.1, AL353804.1, IPO5P1, AP003392.1, AL355574.1 and AC092574.1 were considered as protective IncRNA (HR < 1, B < 0, LASSO coefficient $<$ ) while LINC01705, AP001528.2, AC009948.1, HAGLR and AP001033.2 were risk IncRNA (HR >1, B >0, LASSO coefficient $>0)$. The heatmap showed the Loading [MathJax]/jax/output/CommonHTML/fonts/TeX/fontdata.js group in training set (Fig. 3a), testing set 
(Fig. 3b) and TCGA-STAD dataset (Fig. 3c). As illustrated, the low-risk group tended to express protective IncRNA while the high-risk group were more likely to express risk IncRNA. The correlation between RS and survival time and status in STAD patients were also plotted in the training set (Fig. 3e), testing set (Fig. 3f) and TCGA-STAD dataset (Fig. 3g). In all these datasets, patients with higher RS tended to face a shorter survival time and worse survival status.

Multivariate Cox regression analysis was used to evaluate the independent prognostic indicators. The results of the multivariable analysis showed the RS was a robust and independent prognostic indicator in the training set (Fig. 4a), testing set (Fig. 4b) and TCGA-STAD dataset (Fig. 4c) $(P<0.05)$. Kaplan-Meier analysis was also conducted on the training set (Fig. 4d), testing set (Fig. 4e) and TCGA-STAD dataset (Fig. 4f). Distinctly, there is a strong and significant difference between the two types of risk groups $(P<$ 0.0005). Furthermore, ROC curve was adopted to appraise the accuracy of the model in the training set (Fig. 4g), testing set (Fig. 4h) and TCGA-STAD dataset (Fig. 4i). The area under the curve (AUC) was applied to evaluate the accuracy of the model's prediction in 1-year, 3-year, and 5-year. The value of AUC prompts the model to predict well in prognostic prediction.

\section{Construction of the prognostic mRNA-LncRNA network and identification of involved signaling pathways}

The mRNAs co-expressed with the LncRNAs in the risk score model were obtained by using the correlation test with $\mathrm{R}$ (Pearson correlation coefficient $>0.3, P<0.001$ ). Then all these mRNAs and LncRNAs were used to construct the prognostic mRNA-IncRNA interaction network (Fig. 5a and Fig. 5b). The most significant GO terms for biological process (BP) (Fig. 5c), cellular component (CC) (Fig. 5d), and molecular function (MF) (Fig. 5e), as well as KEGG pathways (Fig. 5f), were analyzed to reveal potential biological functions of the mRNAs in the network (Gene ratio $>0.05, P<0.05$ ). The results showed that the mRNAs in the network were mainly involved in autophagy both in GO and KEGG analysis. GSEA analysis uncovered that the DEGs were significantly enriched in several common signaling pathways (Fig. $5 \mathrm{~g}$ ) (Table 3). 
Table 3

The results of the GSEA analysis

\begin{tabular}{|llll|}
\hline Signaling pathway & NES & NOM $p$-value* & FDR q-value* \\
\hline ECM receptor interaction & 2.05 & 0.00 & 0.05 \\
\hline Neuroactive ligand receptor interaction & 2.02 & 0.00 & 0.03 \\
\hline Complement and coagulation cascades & 1.97 & 0.00 & 0.04 \\
\hline Focal adhesion & 1.89 & 0.01 & 0.04 \\
\hline Calcium signaling pathway & 1.88 & 0.00 & 0.04 \\
\hline Peroxisome & -2.26 & 0.00 & 0.00 \\
\hline Base excision repair & -2.19 & 0.00 & 0.00 \\
\hline Spliceosome & -2.15 & 0.00 & 0.00 \\
\hline Nucleotide excision repair & -2.11 & 0.00 & 0.01 \\
\hline RNA degradation & -2.11 & 0.00 & 0.01 \\
\hline $\begin{array}{l}\text { NES: normalized enrichment score; FDR q-value: false discovery rate q value; NOM } p \text {-value: } \\
\text { normalized } p \text { value; }{ }^{*} \text { FDR q-value }<0.05{ }^{*} \text { NOM } p \text {-value< } 0.05 \text {. }\end{array}$ & \\
\hline
\end{tabular}

\section{Nomograms for personalized prognostic prediction in STAD patients}

A nomogram incorporating the risk type and clinical characters was built to estimate individual survival probability quantitatively of STAD patients. The plot showed the 1-year, 3-year, and 5-year survival probabilities in TCGA-STAD dataset (Fig. 6).

\section{Discussion}

Autophagy is a self-degradative process that plays an essential role in equilibrating energy, eliminating misfolded or aggregated proteins, and reacting to stimuli [30]. To date, three types of autophagy have been found: macroautophagy, microautophagy and chaperone-mediated autophagy [11].

Macroautophagy is considered as the main form of autophagy, which is extensively studied compared to the other two types [31-33]. The process of autophagy is also classified into four critical steps: initiation, nucleation, maturation, and degradation [34]. In the past decades, researchers have discovered 32 autophagy-related genes (Atgs) in yeast, most of which are also highly conserved in mammals significantly [35].

In recent years, some researches have reported that autophagy has a strong relationship between the Loading [MathJax]/jax/output/CommonHTML/fonts/TeX/fontdata.js :lin1 (protein homologue of the yeast ATG6) 
was much overexpressed in malignant tissues than the nonmalignant tissues in GC. What's more, they also found that overexpression of Beclin1 was related with a poor prognosis for GC. Liao et al. [37] discovered that "stone-like" structure pattern of LC3A (an autophagosomal biomarker) was correlated with increased recurrence and worse survival possibility in gastric carcinoma.

As being recognized previously, IncRNAs such as H19 and HOTAIR played a key role as primary regulators in carcinogenesis of GC [38-40]. Until now, numerous studies have proved that IncRNAs were highly active in plenty of pathological processes of GC, such as proliferation and metastasis. Among them, some IncRNAs were defined as protective factors while others were risk factors [25, 41-44]. Furthermore, several studies have proved that IncRNAs participated in the progression, especially malignant progression of GC through regulating autophagy-related mRNAs [24-29, 45].

Although numerous researches have been performed and much is known about IncRNAs and autophagy in GC, previous studies mainly focused on the single IncRNA. The prognostic system relied on the multiple autophagy-related IncRNAs is still not clear. More importantly, as one of the deadliest cancers all over the world, prognosis evaluation of patients with GC still depend too much on the pathological analysis currently, which also facing many challenges and inconvenience in the clinic.

In this study, we obtained the autophagy-related IncRNAs through correlation test of the autophagyrelated genes. The expression of the IncRNAs was profiled from the TCGA-STAD dataset. 24 IncRNAs were found strongly linked with the survival of TCGA-STAD through Kaplan-Meier and univariate Cox regression analysis. We used the LASSO regression analysis to build the model in the training set and found 11 prognostic signatures of IncRNAs. The RS was calculated by integrating IncRNAs expression levels and corresponding LASSO coefficients for each patient. AC005586.1, AL353804.1, IPO5P1, AP003392.1, AL355574.1 and AC092574.1 were considered as protective IncRNA while LINC01705, AP001528.2, AC009948.1, HAGLR and AP001033.2 were risk IncRNA. The accuracy of the model was tested in the testing set, and TCGA-STAD dataset and the RS was found significantly corresponded with patient outcomes in both testing set and TCGA-STAD dataset.

Go analysis revealed that the mRNAs in the prognostic network were mainly involved in the autophagy, which is consistent with the expected results. The MF of GO analysis uncovered that these mRNAs were also have a link with ubiquitin or ubiquitin-like protein ligase binding. Ubiquitin (Ub) is a protein highly conserved in all eukaryotes and bears many potential sites for additional post-translational modifications [46]. Ub was one of the most prominent factors in modifying protein substrates and degradation [47]. The proteolytic system based on ubiquitin and autophagy are two prime systems in eukaryotic cells $[48,49]$. Studies have shown that ubiquitin, as a capital regulator, has participated in all processes in the autophagy flux [50]. Atg8 was a ubiquitin-like protein, which was also found crucial for the autophagosome formation and consisted the lipid conjugation system in autophagy [51].

KEGG analysis uncovered that the mRNAs were primarily involved in the apoptosis, mTOR pathway, p53 pathway and PI3K-Akt pathway. There are two types of autophagy-related signaling pathways. One is Loading [MathJax]/jax/output/CommonHTML/fonts/TeX/fontdata.js Id PI3K/Akt/mTOR pathways, and the other is 
non-mTOR dependent pathways, such as the p53 pathway [52]. The PI3K/AKT/mTOR pathway regulates many biological processes, including autophagy and is frequently activated in various human cancers [53]. The molecular changes in the PI3K/Akt/mTOR signaling pathway were also found could increase the clinical stage and promote the recurrence in carcinoma $[53,54]$. In GC, PI3K/Akt/mTOR activation was found significantly upregulated in GC cells, which results in the inhibition of autophagy of GC cells [55]. Additionally, inhibition of the PI3K/Akt/mTOR pathway increased the autophagic flux and promoted the apoptosis of cancer cells [56].

The results of the GSEA analysis showed that the high-risk group was much active in the ECM receptor interaction. Some studies indicated that autophagy affected the extracellular matrix (ECM), thus participating the invasiveness and metastasis of cancer cells. In a rapidly growing and aggressive tumor, biosynthesis is highly demanded obviously. And in this process, detachment-induced autophagy will help the cancer cells get rid of ECM contact and promoting the metastasis subsequently during advanced cancer stage $[57,58]$. Autophagy will also induce metastatic cancer cells to hibernation if steady connection wasn't established between the new ECM microenvironment and the cancer cells $[59,60]$.

At last, we constructed a nomogram combining the risk type and other clinical features including age, gender, stage and TNM stage, which can predict an individual's clinical outcome quantitatively. By using the nomogram, every patient will get total points based on his or her various indicators respectively. The total points will predict the patient's survival probability in 1, 3-, and 5-year. Obviously, the higher the total points are, the lower the survival probability is.

\section{Conclusion}

In conclusion, we identified a prognostic risk score model consisted of 11 autophagy-related IncRNAs based on TCGA-STAD dataset. This model was an independent predictor and performed well for prognosis in the training set, testing set and TCGA-STAD dataset. A nomogram incorporating the model and clinical feature could accurately predict survival rate for individual GC patients. Our finding suggests that the 11-autophagy IncRNA risk score model may help facilitate individual prediction of patient's prognosis with $\mathrm{GC}$ in the clinic.

\section{Abbreviations}

AUC: area under the curve; BP: biological process; CC: cellular component; ECM: extracellular matrix; DEGs: differentially expressed genes; GC: gastric cancer; GO: Gene Ontology; GSEA: Gene Set Enrichment Analysis; HR: hazard ratio; KEGG: Kyoto Encyclopedia of Genes and Genomes; LASSO: least absolute shrinkage and selection operator; IncRNA: long non-coding RNA; MF: molecular function; ROC: Receiver Operating Characteristic; RS: risk score; STAD: stomach adenocarcinoma; TCGA: The Cancer Genome Atlas. 
Acknowledgements

Not applicable.

\section{Authors' contributions}

CMQ designed the risk score model; CMQ, CW and CB analyzed data; $C M Q$ and $C W$ wrote the manuscript. All authors read and approved the final manuscript.

\section{Funding}

This work was supported by grants from the National Natural Science Foundation of China (NO.81602425), the Natural Science Foundation of Anhui Province (NO.1508085QH152)

\section{Availability of data and materials}

Not applicable.

\section{Ethics approval and consent to participate}

No ethics involved.

\section{Consent for publication}

Written consent was obtained from all participants.

\section{Competing interests}

The authors declare that they have no competing interests.

\section{References}

1. Bray F, Ferlay J, Soerjomataram I, Siegel RL, Torre LA, Jemal A. Global cancer statistics 2018 : GLOBOCAN estimates of incidence and mortality worldwide for 36 cancers in 185 countries. CA Cancer J Clin. 2018;68(6):394-424.

2. Crew KD, Neugut Al. Epidemiology of GC. World J Gastroenterol. 2006;12(3):354-362.

3. Kamangar F, Dores GM, Anderson WF. Patterns of cancer incidence, mortality, and prevalence across five continents: defining priorities to reduce cancer disparities in different geographic regions of the world. J Clin Oncol. 2006;24(14):2137-2150.

4. Bosetti C, Bertuccio P, Malvezzi M, Levi F, Chatenoud L, Negri E, La Vecchia C. Cancer mortality in Europe, 2005-2009, and an overview of trends since 1980. Ann Oncol. 2013;24(10):2657-2671.

5. Fock KM. Review article: the epidemiology and prevention of GC. Aliment Pharmacol Ther. 2014;40(3):250-260. 
7. Jemal A, Bray F, Center MM, Ferlay J, Ward E, Forman D. Global cancer statistics. CA Cancer J Clin. 2011;61(2):69-90.

8. Schmidt N, Peitz U, Lippert H, Malfertheiner P. Missing GC in dyspepsia. Aliment Pharmacol Ther. 2005;21(7):813-820.

9. Verdecchia A, Francisci S, Brenner H, Gatta G, Micheli A, Mangone L, Kunkler I, Group E-W. Recent cancer survival in Europe: a 2000-02 period analysis of EUROCARE-4 data. Lancet Oncol. 2007;8(9):784-796.

10. Mizushima N. Autophagy: process and function. Genes Dev. 2007;21(22):2861-2873.

11. Mizushima N, Komatsu M. Autophagy: renovation of cells and tissues. Cell. 2011;147(4):728-741.

12. Zhao GX, Pan H, Ouyang DY, He XH. The critical molecular interconnections in regulating apoptosis and autophagy. Ann Med. 2015;47(4):305-315.

13. Ren C, Ren T, Yang K, Wang S, Bao X, Zhang F, Guo W. Inhibition of SOX2 induces cell apoptosis and G1/S arrest in Ewing's sarcoma through the PI3K/Akt pathway. J Exp Clin Cancer Res. 2016;35:44.

14. Chang CH, Lee CY, Lu CC, Tsai FJ, Hsu YM, Tsao JW, Juan YN, Chiu HY, Yang JS, Wang CC. Resveratrol-induced autophagy and apoptosis in cisplatin-resistant human oral cancer CAR cells: A key role of AMPK and Akt/mTOR signaling. Int J Oncol. 2017;50(3):873-882.

15. Glick D, Barth S, Macleod KF. Autophagy: cellular and molecular mechanisms. J Pathol. 2010;221(1):3-12.

16. Aita VM, Liang XH, Murty VV, Pincus DL, Yu W, Cayanis E, Kalachikov S, Gilliam TC, Levine B. Cloning and genomic organization of beclin 1, a candidate tumor suppressor gene on chromosome 17q21. Genomics. 1999;59(1):59-65.

17. Liang XH, Jackson S, Seaman M, Brown K, Kempkes B, Hibshoosh H, Levine B. Induction of autophagy and inhibition of tumorigenesis by beclin 1. Nature. 1999;402(6762):672-676.

18. White E. Deconvoluting the context-dependent role for autophagy in cancer. Nat Rev Cancer. 2012;12(6):401-410.

19. Maes H, Rubio N, Garg AD, Agostinis P. Autophagy: shaping the tumor microenvironment and therapeutic response. Trends Mol Med. 2013;19(7):428-446.

20. Maes H, Kuchnio A, Peric A, Moens S, Nys K, De Bock K, Quaegebeur A, Schoors S, Georgiadou M, Wouters $\mathrm{J}$ et al. Tumor vessel normalization by chloroquine independent of autophagy. Cancer Cell. 2014;26(2):190-206.

21. Li W, Zhou Y, Yang J, Li H, Zhang H, Zheng P. Curcumin induces apoptotic cell death and protective autophagy in human GC cells. Oncol Rep. 2017;37(6):3459-3466.

22. Wang X, Wu WKK, Gao J, Li Z, Dong B, Lin X, Li Y, Li Y, Gong J, Qi C et al. Autophagy inhibition enhances PD-L1 expression in GC. J Exp Clin Cancer Res. 2019;38(1):140.

23. Shen YC, Li CP, Yen CJ, Hsu C, Lin YL, Lin ZZ, Chen LT, Su WC, Chao Y, Yeh KH et al. Phase II multicentered study of low-dose everolimus plus cisplatin and weekly 24-hour infusion of high-dose 
5 -fluorouracil and leucovorin as first-line treatment for patients with advanced GC. Oncology. 2014;87(2):104-113.

24. YiRen H, YingCong Y, Sunwu Y, Keqin L, Xiaochun T, Senrui C, Ende C, XiZhou L, Yanfan C. Long noncoding RNA MALAT1 regulates autophagy associated chemoresistance via miR-23b-3p sequestration in GC. Mol Cancer. 2017;16(1):174.

25. Zhu L, Zhu Y, Han S, Chen M, Song P, Dai D, Xu W, Jiang T, Feng L, Shin VY et al. Impaired autophagic degradation of IncRNA ARHGAP5-AS1 promotes chemoresistance in GC. Cell Death Dis. 2019;10(6):383.

26. Zhao Y, Guo Q, Chen J, Hu J, Wang S, Sun Y. Role of long non-coding RNA HULC in cell proliferation, apoptosis and tumor metastasis of GC: a clinical and in vitro investigation. Oncol Rep. 2014;31(1):358-364.

27. Wang LL, Zhang L, Cui XF. Downregulation of long noncoding RNA LINC01419 inhibits cell migration, invasion, and tumor growth and promotes autophagy via inactivation of the PI3K/Akt1/mTOR pathway in GC. Ther Adv Med Oncol. 2019;11:1758835919874651.

28. Yu ZY, Wang Z, Lee KY, Yuan P, Ding J. Effect of silencing colon cancer-associated transcript 2 on the proliferation, apoptosis and autophagy of GC BGC-823 cells. Oncol Lett. 2018;15(3):3127-3132.

29. Shao G, Zhao Z, Zhao W, Hu G, Zhang L, Li W, Xing C, Zhang X. Long non-coding RNA MALAT1 activates autophagy and promotes cell proliferation by downregulating microRNA-204 expression in GC. Oncol Lett. 2020;19(1):805-812.

30. Ravanan P, Srikumar IF, Talwar P. Autophagy: The spotlight for cellular stress responses. Life Sci. 2017;188:53-67.

31. Wen X, Klionsky DJ. An overview of macroautophagy in yeast. J Mol Biol. 2016;428(9 Pt A):16811699.

32. Nishida Y, Arakawa S, Fujitani K, Yamaguchi H, Mizuta T, Kanaseki T, Komatsu M, Otsu K, Tsujimoto Y, Shimizu S. Corrigendum: Discovery of Atg5/Atg7-independent alternative macroautophagy. Nature. 2016;533(7601):130.

33. Munz C. The Macroautophagy Machinery in Endo- and Exocytosis. J Mol Biol. 2017;429(4):473-485.

34. Feng Y, He D, Yao Z, Klionsky DJ. The machinery of macroautophagy. Cell Res. 2014;24(1):24-41.

35. Nakatogawa H, Suzuki K, Kamada Y, Ohsumi Y. Dynamics and diversity in autophagy mechanisms: lessons from yeast. Nat Rev Mol Cell Biol. 2009;10(7):458-467.

36. Qu B, Yao L, Ma HL, Chen HL, Zhang Z, Xie J. Prognostic significance of autophagy-related proteins expression in resected human gastric adenocarcinoma. J Huazhong Univ Sci Technolog Med Sci. 2017;37(1):37-43.

37. Liao W, Sun L, Wang C, Huang H, Liu J, Liao W, Shi M. LC3A-positive "stone-like" structures predict an adverse prognosis of GC. Anat Rec (Hoboken). 2014;297(4):653-662.

38. Cheng C, Qin Y, Zhi Q, Wang J, Qin C. Knockdown of long non-coding RNA HOTAIR inhibits cisplatin resistance of GC cells through inhibiting the PI3K/Akt and Wnt/beta-catenin signaling pathways by Loading [MathJax]/jax/output/CommonHTML/fonts/TeX/fontdata.js 
up-regulating miR-34a. Int J Biol Macromol. 2018;107(Pt B):2620-2629.

39. Endo H, Shiroki T, Nakagawa T, Yokoyama M, Tamai K, Yamanami H, Fujiya T, Sato I, Yamaguchi K, Tanaka $\mathrm{N}$ et al. Enhanced expression of long non-coding RNA HOTAIR is associated with the development of GC. PLoS One. 2013;8(10):e77070.

40. Okugawa Y, Toiyama Y, Hur K, Toden S, Saigusa S, Tanaka K, Inoue Y, Mohri Y, Kusunoki M, Boland $\mathrm{CR}$ et al. Metastasis-associated long non-coding RNA drives GC development and promotes peritoneal metastasis. Carcinogenesis. 2014;35(12):2731-2739.

41. Fanelli GN, Gasparini P, Coati I, Cui R, Pakula H, Chowdhury B, Valeri N, Loupakis F, Kupcinskas J, Cappellesso R et al. LONG-NONCODING RNAs in gastroesophageal cancers. Noncoding RNA Res. 2018;3(4):195-212.

42. Li H, Zhu H, Zhou Y, Wang H, Niu Z, Shen Y, Lv L. Long non-coding RNA MSTO2P promotes the proliferation and colony formation in GC by indirectly regulating miR-335 expression. Tumour Biol. 2017;39(6):1010428317705506.

43. Ma Y, Liu L, Yan F, Wei W, Deng J, Sun J. Enhanced expression of long non-coding RNA NEAT1 is associated with the progression of gastric adenocarcinomas. World J Surg Oncol. 2016;14(1):41.

44. Guo X, Xia J, Deng K. Long non-coding RNAs: emerging players in GC. Tumour Biol. 2014;35(11):10591-10600.

45. Chen JF, Wu P, Xia R, Yang J, Huo XY, Gu DY, Tang CJ, De W, Yang F. STAT3-induced IncRNA HAGLROS overexpression contributes to the malignant progression of GC cells via mTOR signalmediated inhibition of autophagy. Mol Cancer. 2018;17(1):6.

46. Komander D, Rape M. The ubiquitin code. Annu Rev Biochem. 2012;81:203-229.

47. Zheng N, Shabek N. Ubiquitin Ligases: Structure, Function, and Regulation. Annu Rev Biochem. 2017;86:129-157.

48. Ciechanover A. The unravelling of the ubiquitin system. Nat Rev Mol Cell Biol. 2015;16(5):322-324.

49. Ciechanover A, Kwon YT. Protein Quality Control by Molecular Chaperones in Neurodegeneration. Front Neurosci. 2017;11:185.

50. McEwan DG, Dikic I. The Three Musketeers of Autophagy: phosphorylation, ubiquitylation and acetylation. Trends Cell Biol. 2011;21(4):195-201.

51. Nakatogawa H, Ichimura Y, Ohsumi Y. Atg8, a ubiquitin-like protein required for autophagosome formation, mediates membrane tethering and hemifusion. Cell. 2007;130(1):165-178.

52. Cao Y, Luo Y, Zou J, Ouyang J, Cai Z, Zeng X, Ling H, Zeng T. Autophagy and its role in GC. Clin Chim Acta. 2019;489:10-20.

53. Aoki M, Fujishita T. Oncogenic Roles of the PI3K/AKT/mTOR Axis. Curr Top Microbiol Immunol. 2017;407:153-189.

54. Morgan TM, Koreckij TD, Corey E. Targeted therapy for advanced prostate cancer: inhibition of the PI3K/Akt/mTOR pathway. Curr Cancer Drug Targets. 2009;9(2):237-249. 
55. Li Y, Liu Y, Shi F, Cheng L, She J. Knockdown of Rap1b Enhances Apoptosis and Autophagy in GC Cells via the PI3K/Akt/mTOR Pathway. Oncol Res. 2016;24(5):287-293.

56. Saiki S, Sasazawa Y, Imamichi Y, Kawajiri S, Fujimaki T, Tanida I, Kobayashi H, Sato F, Sato S, Ishikawa $\mathrm{K}$ et al. Caffeine induces apoptosis by enhancement of autophagy via $\mathrm{PI3K} /$ Akt/mTOR/p70S6K inhibition. Autophagy. 2011;7(2):176-187.

57. Guadamillas MC, Cerezo A, Del Pozo MA. Overcoming anoikis-pathways to anchorage-independent growth in cancer. J Cell Sci. 2011;124(Pt 19):3189-3197.

58. Lock R, Debnath J. Extracellular matrix regulation of autophagy. Curr Opin Cell Biol. 2008;20(5):583588.

59. Su Z, Yang Z, Xu Y, Chen Y, Yu Q. Apoptosis, autophagy, necroptosis, and cancer metastasis. Mol Cancer. 2015;14:48.

60. Lu Z, Luo RZ, Lu Y, Zhang X, Yu Q, Khare S, Kondo S, Kondo Y, Yu Y, Mills GB et al. The tumor suppressor gene $\mathrm{ARHI}$ regulates autophagy and tumor dormancy in human ovarian cancer cells. $\mathrm{J}$ Clin Invest. 2008;118(12):3917-3929.

\section{Supplementary Figure Legend}

Supplementary Fig Kaplan-Meier survival curves for TCGA-STAD dataset. Kaplan-Meier survival analysis regarding prime clinical features in GC. ${ }^{*} P<0.05$

\section{Figures}




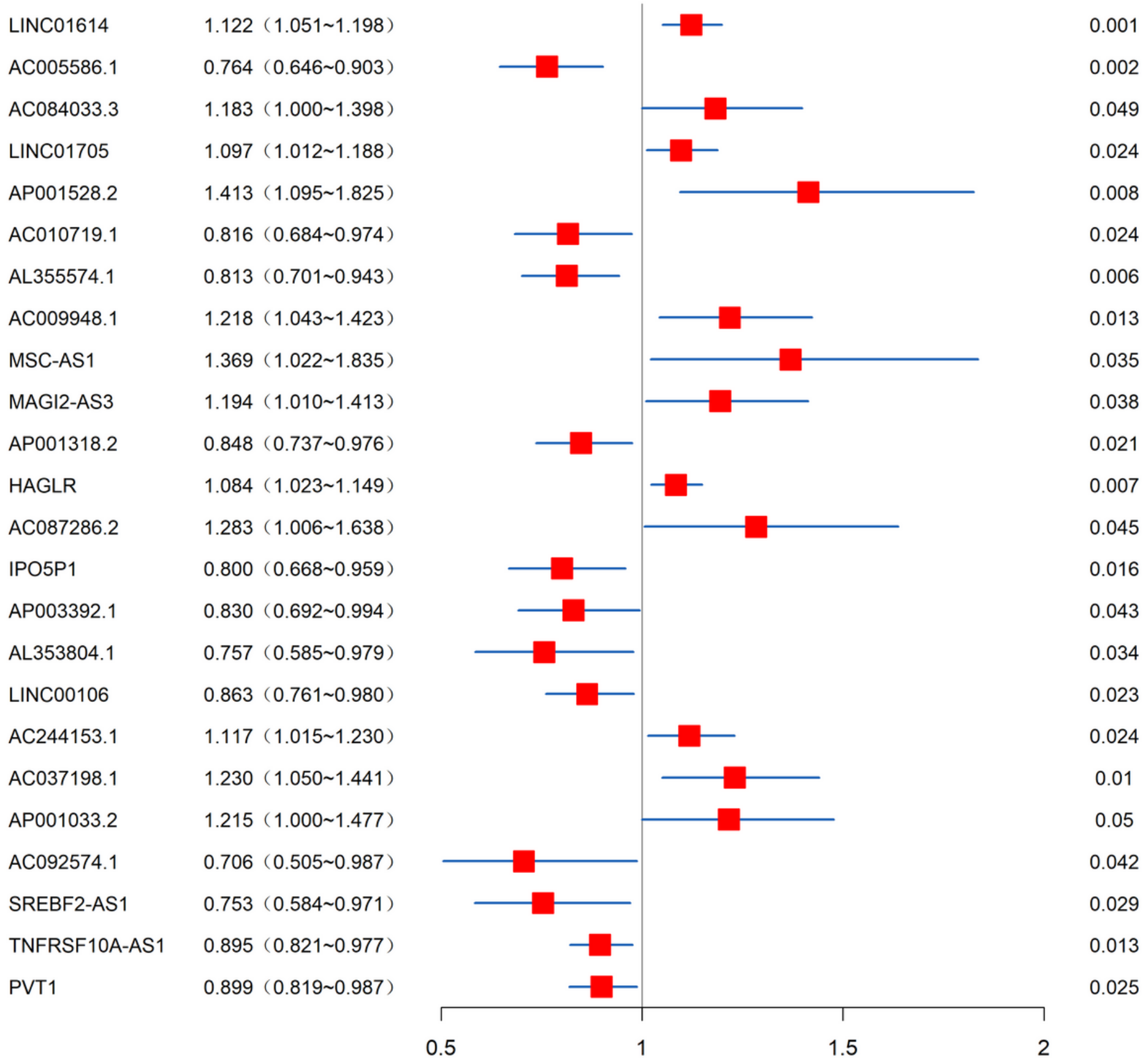

\section{Figure 1}

Screening of the autophagy-related IncRNAs linked with the survival in GC. Forest plot of prognostic autophagy-related IncRNAs in TCGA-STAD dataset based on Kaplan-Meier and univariate Cox regression analysis. ${ }^{*} \mathrm{P}<0.05$ 
a

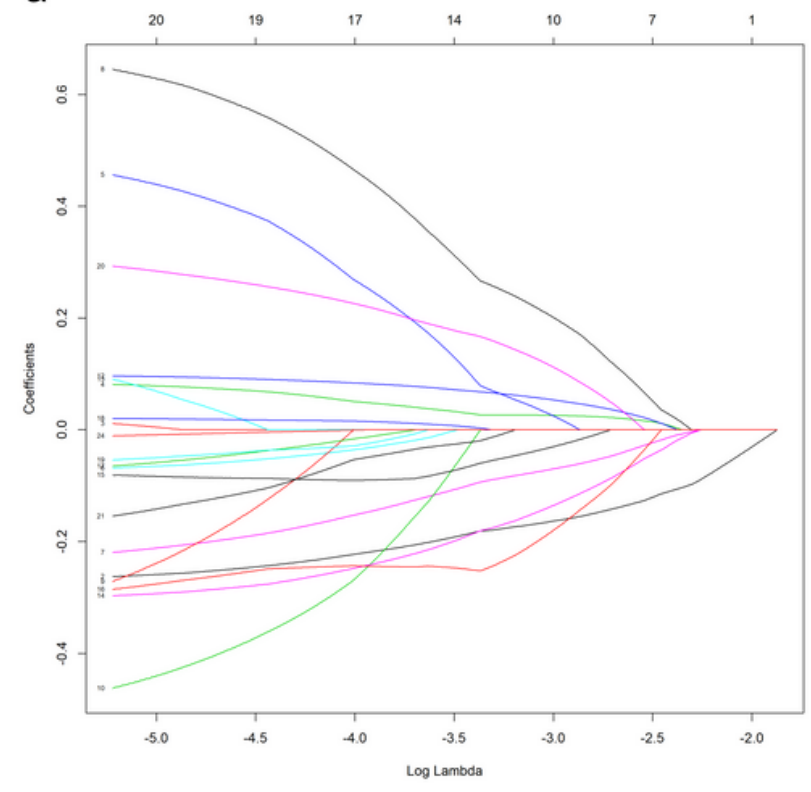

b

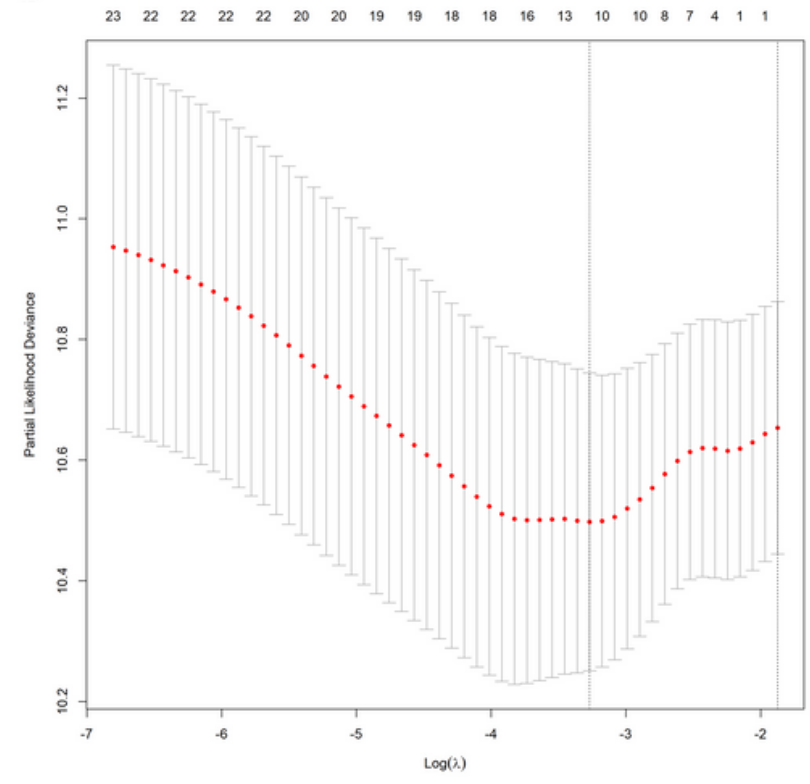

Figure 2

Construction of prognostic risk score model based on LASSO regression analysis. a Coefficient profile of each 24 prognostic autophagy-related IncRNA in the training set. b Selection of the optimal panel (lambda) for the model in the training set.

a

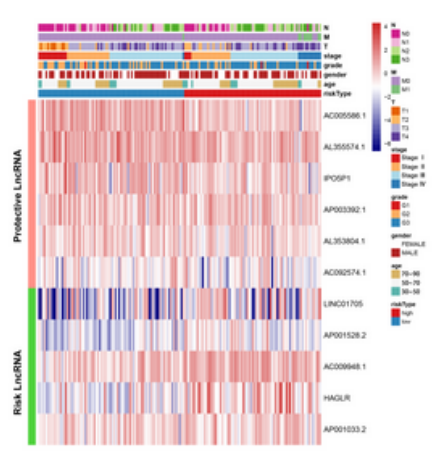

d
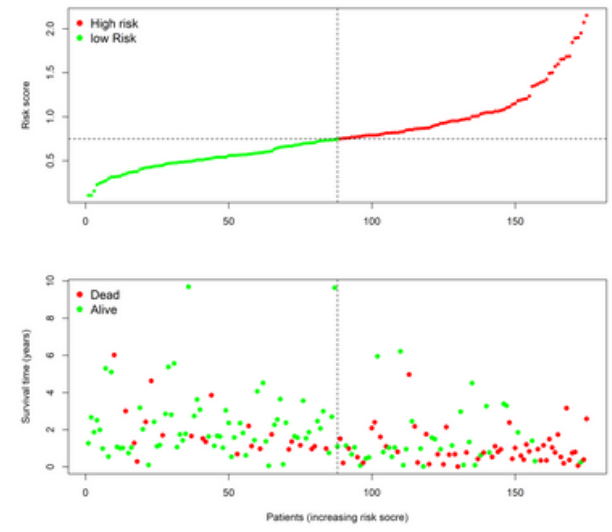

b
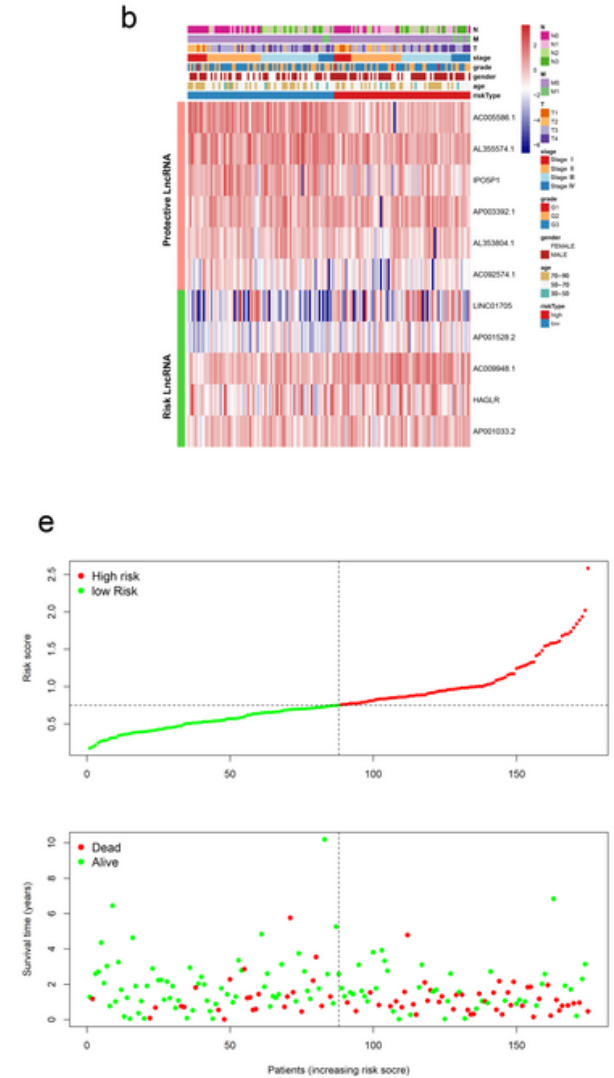
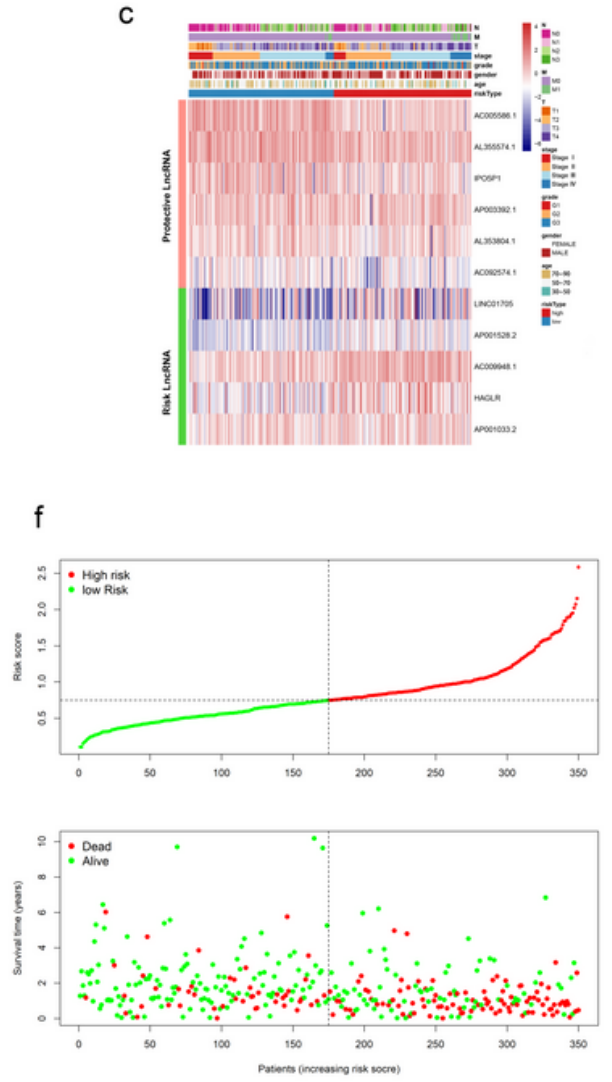


\section{Figure 3}

The link between clinical data and risk score a-c Heatmap plot for the IncRNAs in the model in the training set, testing set and TCGA-STAD dataset. d-e The relationship between the RS and survival time as well as status in the training set, testing set and TCGA-STAD dataset.

a

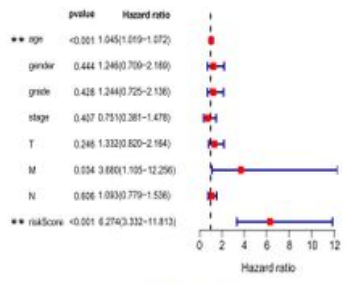

d

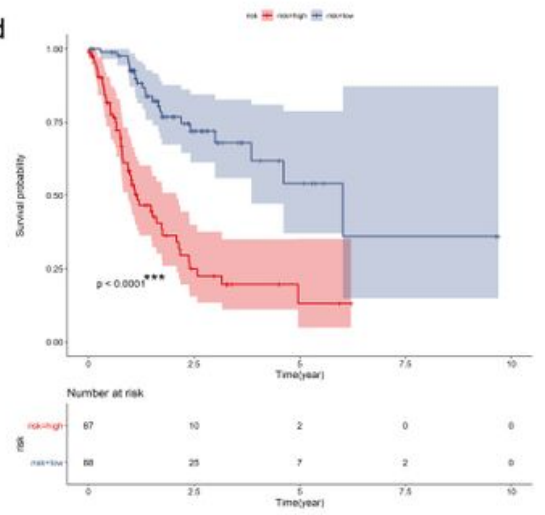

g

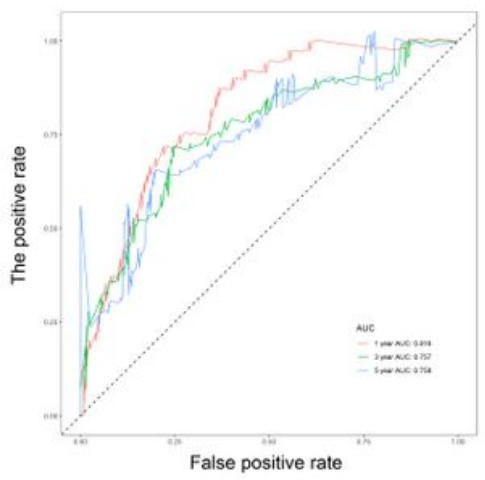

b

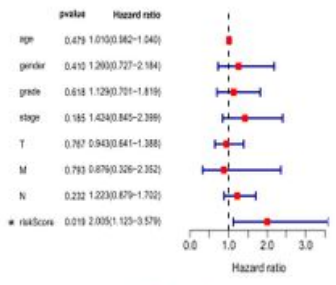

e
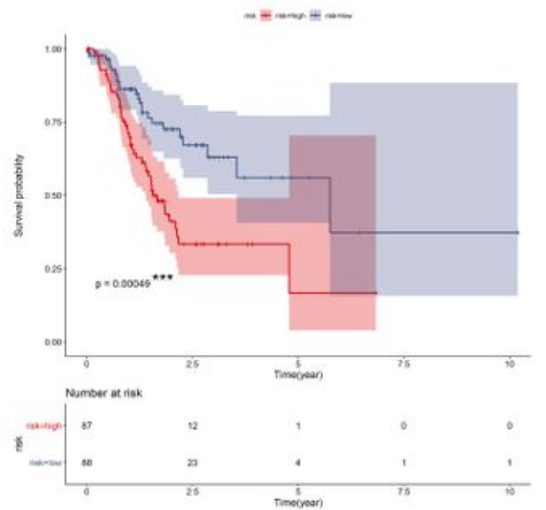

$\mathrm{h}$

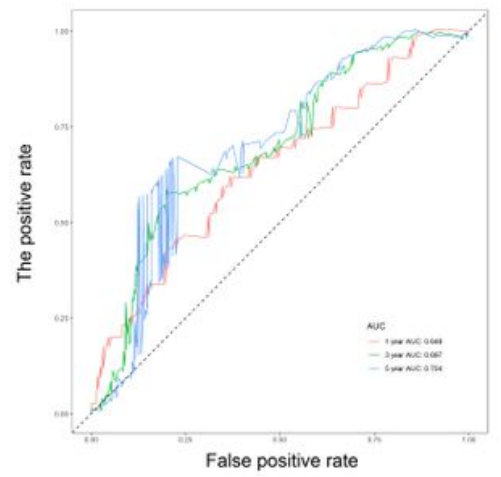

c

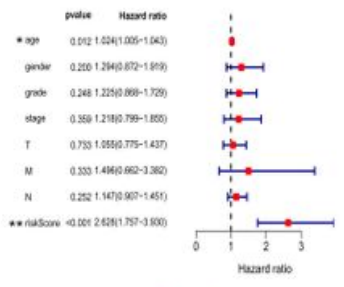

f
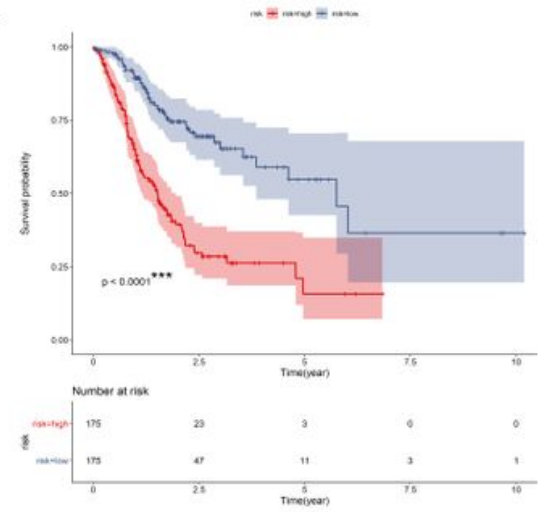

i

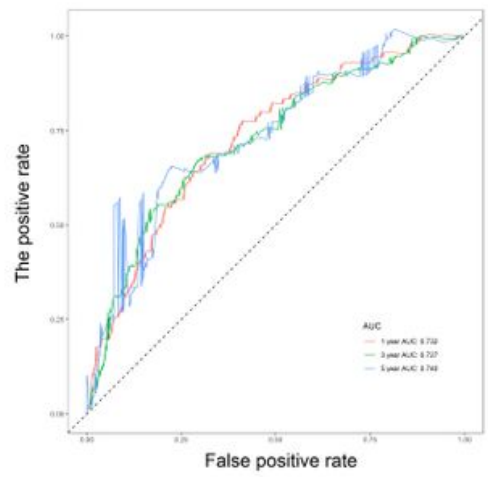

Figure 4

The risk score model performed well for survival prediction. a-c The RS was an independent prognostic predictor in the training set, testing set and TCGA-STAD dataset. d-f Kaplan-Meier analysis based on risk type in the training set, testing set and TCGA-STAD dataset. g-i The ROC curve and the value of AUC in 1year, 3-year, and 5-year in the training set, testing set and TCGA-STAD dataset. ${ }^{*} P<0.05$, ${ }^{\star * P}<0.001$, $* \star * P$ $<0.0005$ 

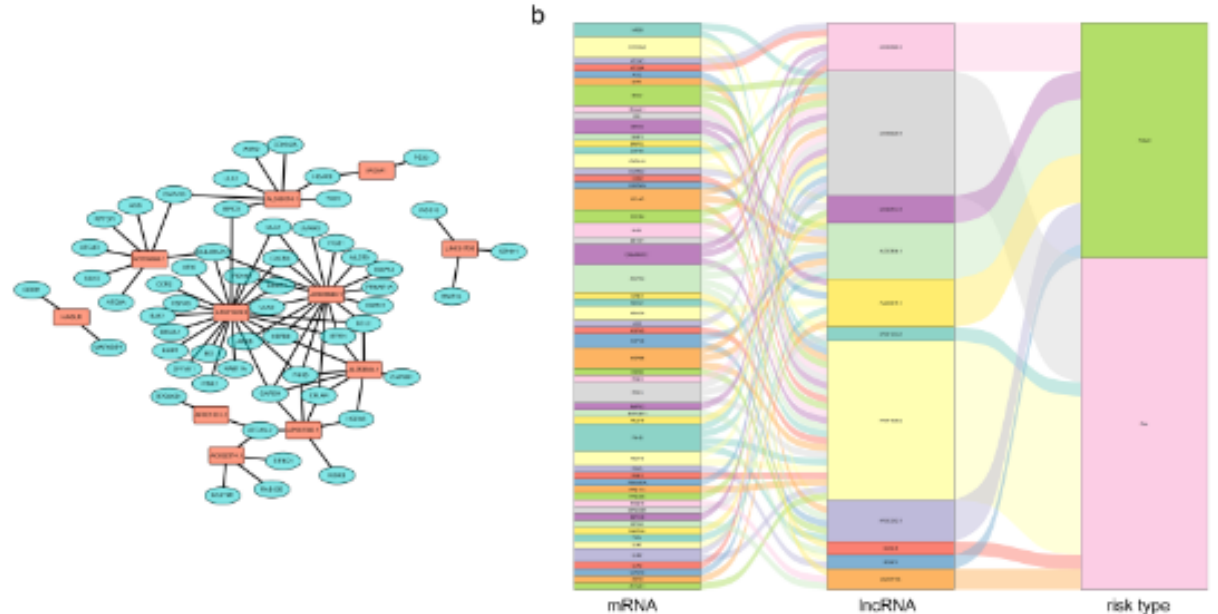

c

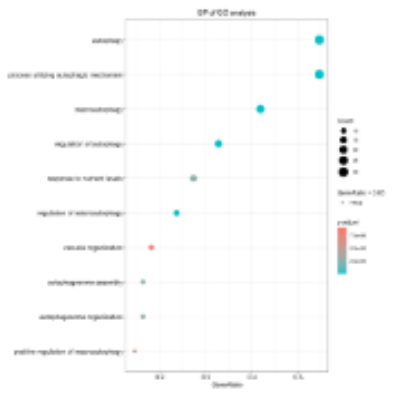

d
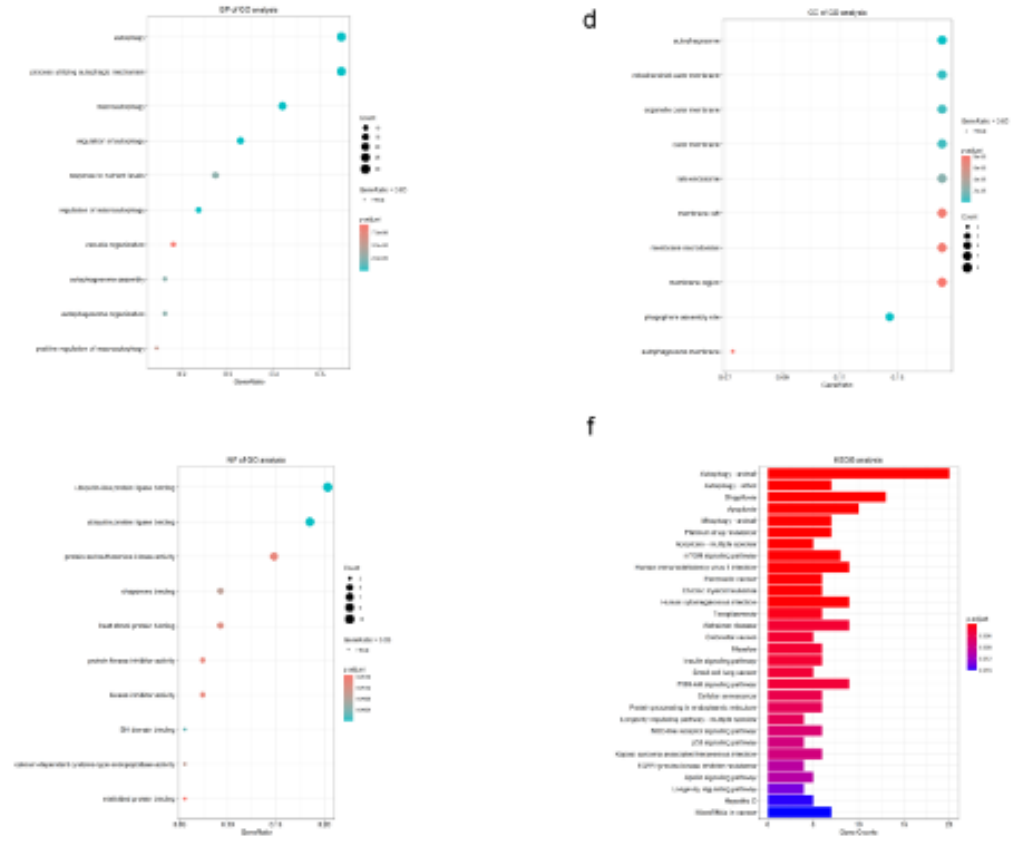

f

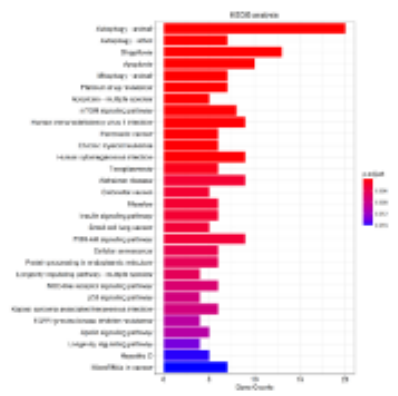

g

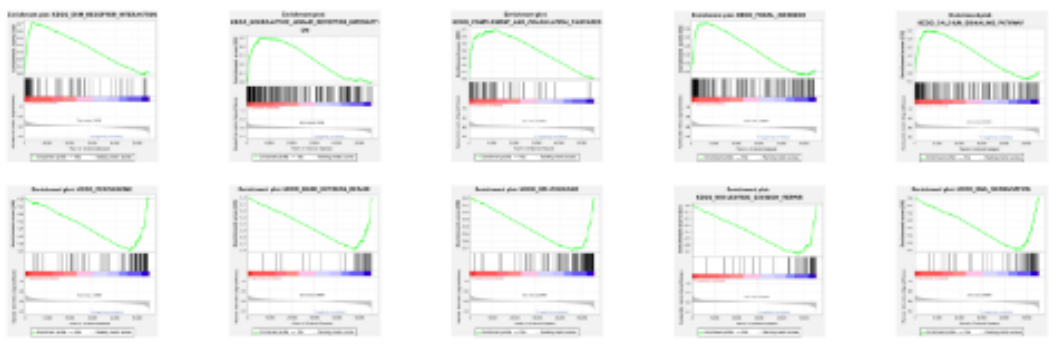

\section{Figure 5}

Construction of the prognostic mRNA-LncRNA interaction network and identification of involved signaling pathways. a The prognostic network based on the IncRNAs in the risk score model and the co-expressed mRNAs. b The Sankey diagram based on the prognostic network. c-e BP, CC, and MF of GO analysis for the mRNAs in the network. f KEGG analysis of the mRNAs in the network. g GSEA analysis of the DEGs 


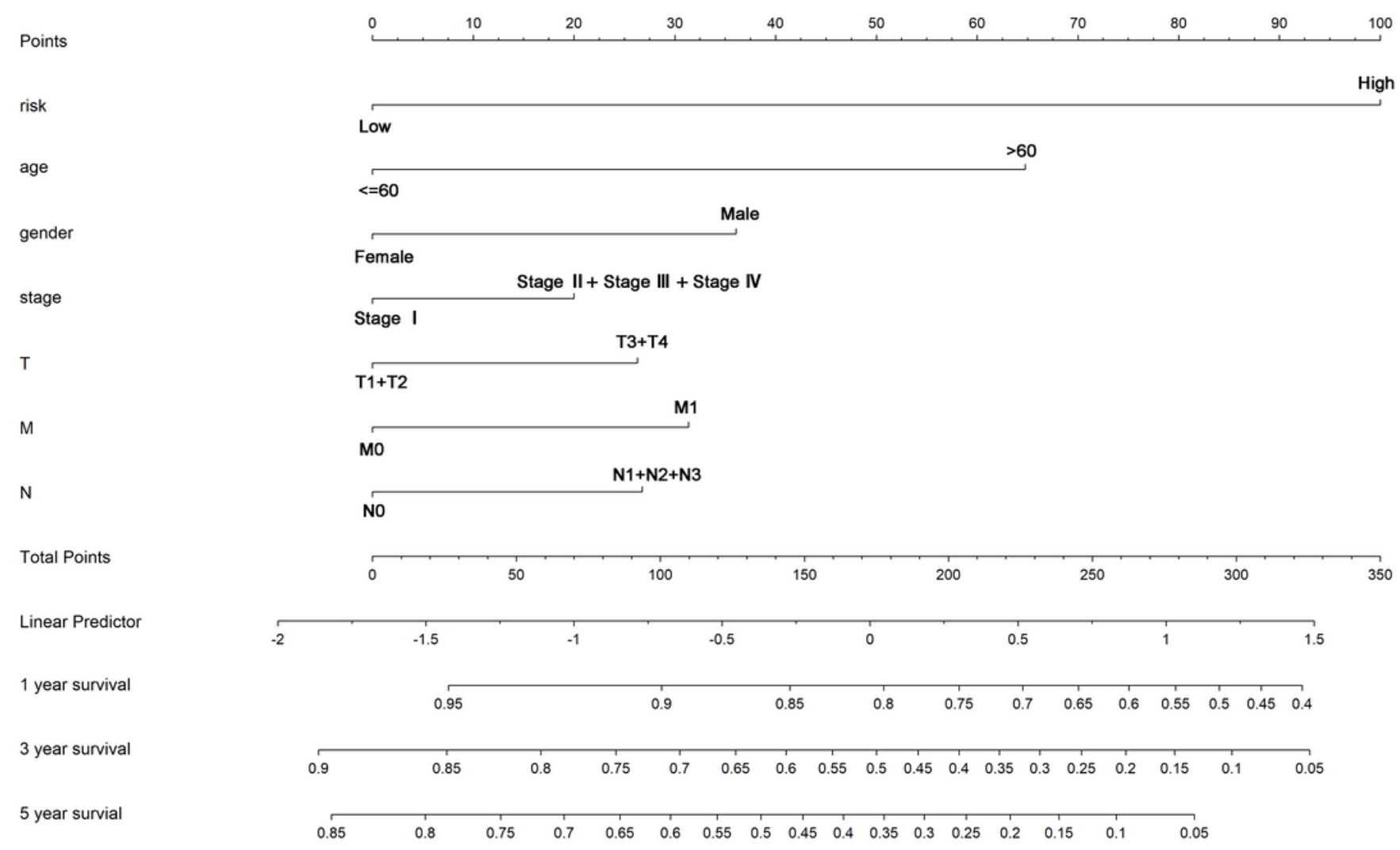

Figure 6

The nomogram for predicting an individual's survival rates in TCGA-STAD dataset. The nomogram is incorporated with the risk type and clinical features to predict personalized survival probabilities in 1-year, 3-year, and 5-year.

\section{Supplementary Files}

This is a list of supplementary files associated with this preprint. Click to download.

- SupplementaryFig.tif 